\title{
An Adaptive Energy Efficient and Reliable Gossip Routing Protocol For Mobile Adhoc Networks
}

\author{
S.Rajeswari and Dr.Y.Venkataramani
}

\begin{abstract}
The current ad hoc network routing protocols require all the nodes to be awake and keep listening which results in waste of energy. The existing energy efficient routing protocols and topology management schemes increase the computing complexity and acquire extra overhead or equipments. To over come these drawbacks, in this paper, we design an Adaptive Energy Efficient and Reliable Gossip Routing (AEERG) Protocol for Mobile Ad hoc Networks. The major objective of the protocol is to achieve energy efficiency and reliability. In this protocol, we set a counter to represent the current number of neighbors at each node which are kept in active state. The value of the counter is adaptively adjusted based on the packet delivery ratio. This results in less energy consumption and reliability in the network-wide communication. By simulation results, we show that the proposed protocol achieves good delivery ratio and high throughput with less packet drop and energy consumption.
\end{abstract}

Index Terms-Delivery ratio, energy consumption, Mobile Adhoc Networks, Reliability, Reliable Gossip Routing (AEERG) Protocol.

\section{INTRODUCTION}

\section{A. Mobile Ad hoc Networks}

A mobile ad-hoc network (MANET) is a collection of many mobile nodes with no infrastructure. To form a network over radio links, the mobile nodes are self-organized. Extending mobility into the self-organized, mobile and wireless domains is the main objective of MANETs where a set of nodes form the network routing infrastructure in an ad-hoc fashion. MANETs are used in those areas where wired network is unavailable and where rapid deployment and dynamic reconfiguration are necessary. These include military battlefields, emergency search, rescue sites, classrooms and conventions, where the participants share information dynamically using their mobile devices [1].

\section{B. Existing routing protocols}

Generally the existing routing protocols are either

1) Table-driven (proactive) routing protocol or

2) On-demand (reactive) routing protocol

S.Rajeswari, Assistant Professor, Department of ECE, Saranathan College of Engineering, Trichy. (e-mail: rajee_ravi@sify.com)

Dr. Y.Venkataramani, Director (Academics), Saranathan college of Engineering, Trichy. (e-mail:diracads@saranathan.ac.in)

\section{Proactive MANET protocol (PMP)}

A proactive MANET protocol (PMP) detects the layout of the network which is active. PMP maintains a routing table at every node.

From the routing table, a route can be determined with minimal delay. The PMP can provide good reliability and low latency. This protocol cannot update the route information immediately for a node moving with high speed. Also for a node moving occasionally, updating the unchanged entry continuously in the routing table results in much traffic overhead and wastage of network resources. PMP is not appropriate for large scale MANETs [2]. PMP is used in DSDV [11], OLSR [14].

\section{Reactive MANET protocol (RMP)}

In Reactive MANET protocol (RMP) when the source node request to communicate with the other node, only then a route between a pair of nodes is found. For nodes with high mobility and for nodes which transmit data occasionally, this on-demand approach is quite suitable. But in RMP the disadvantage is, the source node broadcasts route requests throughout the network and has to wait for the response. This route discovery procedure results in a major delay [2]. RMP is used in, DSR[12], AODV [13] and TORA[15].

\section{E. Gossip Routing Protocol}

For location discovery or for secure routing applications, most ad hoc routing algorithms depend on broadcast flooding. Though flooding is a robust algorithm, because of its extreme redundancy, it is unfeasible in dense networks. The use of flooding algorithms may lead to broadcast storms in large wireless networks where the number of collisions is so high it could cause system failure. Since the packet retransmission is based on the outcome of coin tosses, Gossip [3] is a probabilistic algorithm. The main objective of gossip is to minimize the number of retransmissions, while maintaining the main benefits of flooding.

\section{F. Motivation and Objective}

Optimizing energy consumption in these networks has given high priority, since most of the mobile hosts are not connected to a power supply and battery recharging is tough. Even if there is no traffic or heavy traffic (neighbor nodes are totally redundant for each other), the traditional ad hoc routing protocols necessitate all nodes to continue listening, thereby wasting the energy. Hence this reduces the lifetime of 
the nodes as well as the network's lifetime [3].The major objective as proposed in (GSP) is to achieve energy efficiency by putting some nodes in a sleep mode. The potential disadvantage of this approach is that packets may go through longer paths if the nodes sleeping are on the shortest paths between source and destination nodes, resulting in more energy consumption in the network-wide communication. Also, paths will be broken more often due to mode change of the nodes. Therefore, more overhead is generated to overcome the path failures and this will consume some extra energy. So we are concerned if the energy saved by GSP is larger than the extra energy, consumed by non-optimal paths and extra routing overhead. In addition, sleeping of nodes results in decrease of the network throughput and increase of end to end delay. Hence both energy consumption and reliability can not be achieved. The major objective of [9] is to achieve energy efficiency and reliability. But in this work, since all the nodes are kept in active state, it results in energy wastage.

The existing energy efficient routing protocols and topology management schemes increase the computing complexity and acquire extra overhead. In this paper, based on the gossip-based ad hoc routing, we propose an Adaptive Energy Efficient and Reliable Gossip Routing Protocol to achieve energy efficiency and reliability in mobile ad hoc networks, to overcome the above discussed drawbacks. In this protocol, the nodes can be in active mode with probability $\mathrm{p}$ or sleep mode with probability 1-p which is fixed at the initial stage. We set a counter $B$ to adapt the number of neighbors to which a packet is forwarded. B represents the current number of neighbors at each node which are kept in active state. The value of B is adaptively adjusted based on the packet delivery ratio. This results in more energy consumption and reliability in the network-wide communication.

\section{RELATED WORK}

Xiaobing Hou and David Tipper [3] propose a novel energy saving scheme, termed the Gossip-based Sleep Protocol (GSP). With GSP, each node randomly goes to sleep for some time with gossip sleep probability $p$. When the value of $p$ is small enough, the network stays connected. Mike Burmester, Tri Van Le and Alec Yasinsac [4] consider ways to reduce the number of redundant transmissions in flooding while guaranteeing security. They present several new gossip protocols among that the last two gossip protocols that they have given are fully adaptive, i.e., protocols automatically correct all faults and guarantee delivery.

Yuval Shavitt and Amir Shay [5] introduce the Gossip Network model where travelers can obtain information about the state of dynamic networks by gossiping with peer travelers using ad-hoc communication. Travelers then use the gossip information to re-choose their path and find the shortest path to destination. Zhongmin Shi and Hong Shen [6] Gossip-based techniques recently adopted in mobile ad hoc network (MANET) system have achieved significant improvement on network overhead, routing efficiency and reliability. Zygmunt J. Haas, Joseph Y. Halpern, and Li
(Erran) Li [7], propose a gossiping-based approach, where each node forwards a message with some probability, to reduce the overhead of the routing protocols. With less number of executions maximum of nodes get the message that depends on the gossiping probability and the topology of the network.

Hany Morcos Ibrahim Matta Azer Bestavros [8] propose a new approach - by control-theoretic adaptations similar to those widely used in the Internet, e.g. additive-increase multiplicative-decrease (AIMD) of TCP for reacting to congestion conditions. Matthew J. Miller Cigdem Sengul Indranil Gupta [9] propose networking protocols for multi-hop wireless sensor networks (WSNs) which are required to simultaneously minimize resource usage as well as optimize performance metrics such as latency and reliability. They explore the energy-latency-reliability trade-off for broadcast in multi-hop WSNs, by presenting a new protocol called PBBF (Probability-Based Broadcast Forwarding). Xiang-yang li, Kousha Moaveninejad and Ophir Frieder [10] used Gossip based routing method and re-checked to reduce the number of messages in both wired networks and wireless ad hoc networks.

\section{GOSSIP ROUTING IN AD HOC NETWORKS}

Flooding is a basic element in many of the ad hoc routing protocols. But the use of flooding algorithms may lead to broadcast storms in large wireless networks, where the number of collisions is so high, causing system failure. Since the packet retransmission is based on the outcome of coin tosses, the main objective of gossip is to minimize the number of retransmissions, while maintaining the main benefits of flooding.

A message is normally transmitted as a broadcast rather than a unicast communication in adhoc networks. So the message is received by the entire nodes one hop away from the sender. Since wireless resources are expensive, it will be better if we use this physical-layer broadcasting feature of the radio transmission. In the gossiping protocol, we control the probability with which this physical-layer broadcast is sent [7].

The basic gossiping protocol is simple. A source sends a route request with probability 1 . When a node first receives a route request, with probability $\mathrm{p}$ it broadcasts the request to its neighbors and with probability 1-p it discards the request; if the node receives the same route request again, it is discarded. Thus, a node broadcasts a given route request at most once [6]. Thus, in almost all executions of the algorithm, either scarcely any nodes receive the message, or most of them do. Ideally, we could make less number of executions where the gossip dies out relatively low while also keeping the gossip probability low, to reduce the message overhead [7].

The gossip routing protocol satisfies the following conditions:

1) The main portion of the protocol involves periodic, pairwise, inter-process communications.

2) During these communications the information exchanged is of bounded size.

3) When agents interact, just to intimate the state of the 
other agent(at least the change in the state of one agent)

4) A gossip communication does not occur when A pings $\mathrm{B}$, to compute the response time, as this does not involve the transmission between agents.

5) Reliable communication is not implicit.

6) The protocol costs are insignificant since the frequency of the communications is low compared to classic message latencies.

As we mentioned earlier, the current ad hoc network routing protocols require all the nodes to be awake and keep listening. This wastes a lot of energy,

\section{ADAPTIVE ENERGY EFFICIENT AND RELIABLE GOSSIP (AEERG) ROUTING PROTOCOL}

Our observation is that if gossiping can make all the nodes receive a message, then the nodes forwarding the message are connected at least by the paths the message passes through. Therefore, in a static network without mobility (e.g., a sensor network), with certain probability $p^{\prime}$, gossiping protocols [7] can make almost all nodes in the network receive the message. Then if all nodes go to sleep with probability $p=\left(1-p^{\prime}\right)$, almost all the awake nodes stay connected. Thus, we can safely put a percentage $(p)$ of the nodes in sleep mode without losing network connectivity. We term $p$ the gossip sleep probability.

Let us assume that every node in the ad hoc network chooses an equally distributed random time interval, known as gossip interval. When the time is up, the node will immediately choose another random interval independently. To make it possible, we assume the feasible maximum gossip interval is much smaller than the lifetime of the network

1) Each node independently generates a random time interval and chooses either going to sleep with probability $p$ or staying awake with probability $1-p$ for the interval.

2) Every sleeping node wakes up at the end of its interval

3) Every node repeats the above process for every random interval independently

A node (which wants to communicate) maintains a control variable called $\mathrm{B}$ which represents the current number of neighbors at each node which are kept in active state. The rest of the nodes will be in either $p$ or $1-p$ state. The higher $-\mathrm{B}$ is the more power the node uses to send packets and thus the communication is more reliable. When node $X$ needs to broadcast a data packet, $X$ looks up its neighbor list for the distance between itself and its neighbors numbered B. $X$ then calculates the amount of power needed to send the packet to that neighbor.

Every node initializes B to one. This means that a node initially broadcasts data packets only to its closest neighbor, thus requiring the least power. After sending data packet, node $X$ waits for a feedback from destination. While receiving packets at the destination, the delivery ratio $\mathrm{D}$ is calculated and it will be sent as a feedback to the source. If $X$ hears a feedback D for the data packet below a reliability threshold RT, $X$ increases the value of B there by increasing the probability of active nodes. This assures the increased delivery ratio. When $\mathrm{D}$ becomes greater than or equal to RT, the value of $B$ is decreased adaptively to decrease the number of forwarding nodes and there by decrease the probability of active nodes. This process continues until either $X$ hears a feedback for the packet or the value of B reaches reliability threshold RT, which is determined by the total number of neighbors. Upon receiving a feedback, $X$ starts to decrease the value of B (after a certain number of acknowledged data packets) to a minimum value of one.

Algorithm:

1) Let sleep probability $P(s)=p$ and awake probability $A(s)=1-p$.

2) Let initial value of $B-1$.

3) $X$ broadcasts data packets to $Y$.

4) At $Y$, calculate delivery ratio, $D=$ Number of packets received /Number of packets sent

5) $Y$ sends D as a feedback to $X$.

6) At $X$, If $D<R T$ then, 6.1. $B=B+\delta$, where $\delta$ is the scale factor. 6.2. Repeat from 3 .

7) Else. If $D \geq R T$, then,

$$
\begin{aligned}
& \text { 7.1. If } B>1 \text {, then, } \\
& \text { 7.1.1. } B=B-\delta \\
& \text { 7.1.2. Repeat from } 3 \text {. } \\
& \text { 7.2. End If }
\end{aligned}
$$

8) End If

9) Repeat from 3.

To summarize, AEERG routing protocol has the following salient features:

1) Unlike existing routing schemes, AEERG is neither single-path nor multi-path; rather each node exploits the multiplicity of paths based on its observed loss conditions.

2) Under AEERG, only for low packet delivery ratios, a node uses high-powered transmissions to reach farther neighbors. For high packet delivery ratios, a node adapts to low-powered transmissions. Thus, AEERG sensibly consumes power based on local error conditions, which maximizes the lifetime of the network and minimizes the cost of the power consumed per successfully delivered data.

3) AEERG aggressively probes for possible routes to deliver data packets, thus reacting quickly within unreliable areas of the network.

\section{SimULATION RESUltS}

\section{A. Simulation Model and Parameters}

NS2 [16] is used to simulate the proposed algorithm. In our simulation, the channel capacity of mobile hosts is set to the same value: $2 \mathrm{Mbps}$. For the MAC layer protocol the distributed coordination function (DCF) of IEEE 802.11 (for wireless LANs) is used. It has the functionality to notify the network layer about link breakage.

In the simulation, mobile nodes move in a 500 meter $x 500$ meter region for 50 seconds simulation time. The number of 
mobile nodes is kept as 40 . We assume each node moves independently with the same average speed. All nodes have the same transmission range of 250 meters. In our simulation, the speed is set as $20 \mathrm{~m} / \mathrm{s}$. The simulated traffic is Constant Bit Rate (CBR). The pause time of the mobile node is kept as 10 sec.

Our simulation settings and parameters are summarized in table I.

TABLE I

SIMULATION PARAMETERS

\begin{tabular}{|l|l|}
\hline No. of Nodes & 40 \\
\hline Area Size & 500 X 500 \\
\hline Mac & 802.11 \\
\hline Radio Range & $250 \mathrm{~m}$ \\
\hline Simulation Time & $50 \mathrm{sec}$ \\
\hline Traffic Source & CBR \\
\hline Rate & $\begin{array}{l}100,200,300,400 \text { and } 500 \\
\mathrm{~kb}\end{array}$ \\
\hline Mobility Model & Random Way Point \\
\hline Speed & $20 \mathrm{~m} / \mathrm{s}$ \\
\hline Pause time & $10 \mathrm{sec}$ \\
\hline Transmit Power & $0.660 \mathrm{w}$ \\
\hline Receiving Power & $0.395 \mathrm{w}$ \\
\hline Idle Power & $0.035 \mathrm{w}$ \\
\hline Initial Energy & $15.1 \mathrm{~J}$ \\
\hline Pvalue & $0.0,0.1,0.2,0.3$ and 0.4 \\
\hline
\end{tabular}

\section{B. Performance Metrics}

Our proposed Adaptive Energy Efficient and Reliable Gossip Routing (AEERG) protocol is compared with Probability Based Broadcast Forwarding (PBBF) [9] protocol. The evaluation is mainly based on performance according to the following metrics:

Throughput: It is the number of packets received successfully.

Average Energy: It is the average energy consumption of all nodes in sending, receiving and forward operations.

Drop: It is the number of packets dropped.

Packet Delivery Fraction: It is the ratio of the fraction of packets received successfully and the total number of packets sent.

\section{Results}

1) Based on Flow (number of neighbor nodes)

In our initial experiment, we vary the number of flows as 1 , 2, 3 and 4.

Fig 1 gives the throughput of protocols when the number of flow is increased. As we can see from the figure, the throughput is more in the case of AEERG than PBBF.

Fig 2 shows the results of energy consumption for the number of flows $1,2,3,4$. From the results, we can see that AEERG scheme has less energy than PBBF scheme, since it has the energy efficient routing.

From Fig 3, we can ensure that the packets dropped are less for AEERG when compared with PBBF.

Fig 4 presents the packet delivery ratio of both the protocols. Since the packet drop is less and the throughput is more, AEERG achieves good delivery ratio, compared to PBBF protocol.

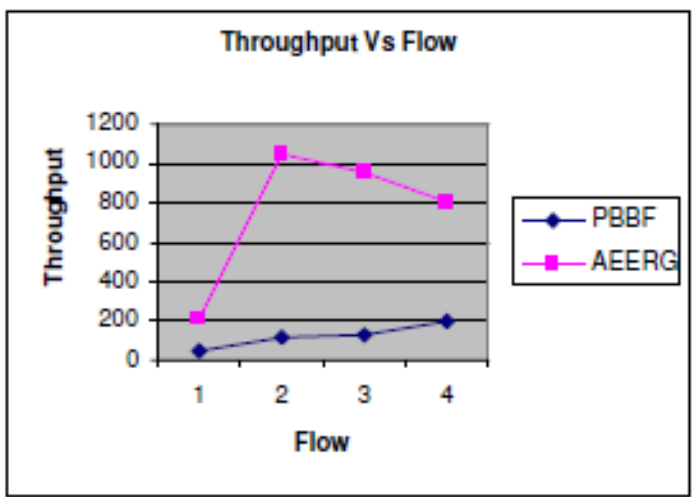

Fig.1 Throughput Vs Flow

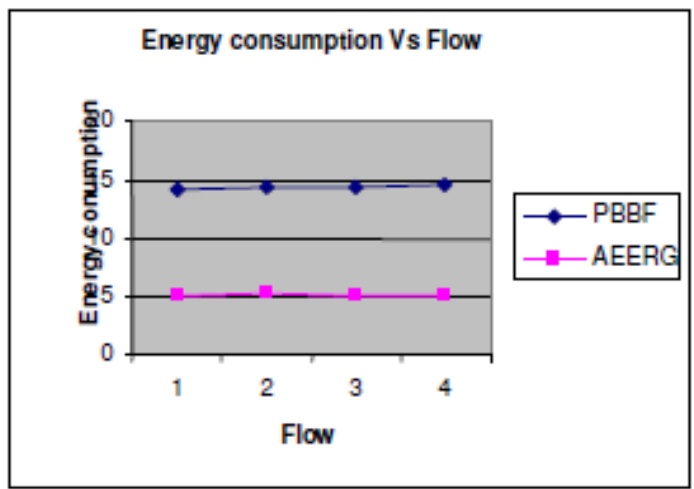

Fig. 2 Energy consumption Vs Flow

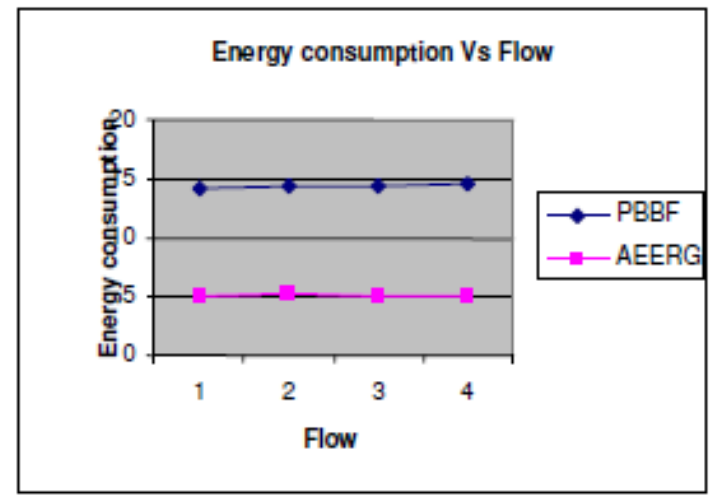

Fig. 3 Energy Consumption Vs Flow

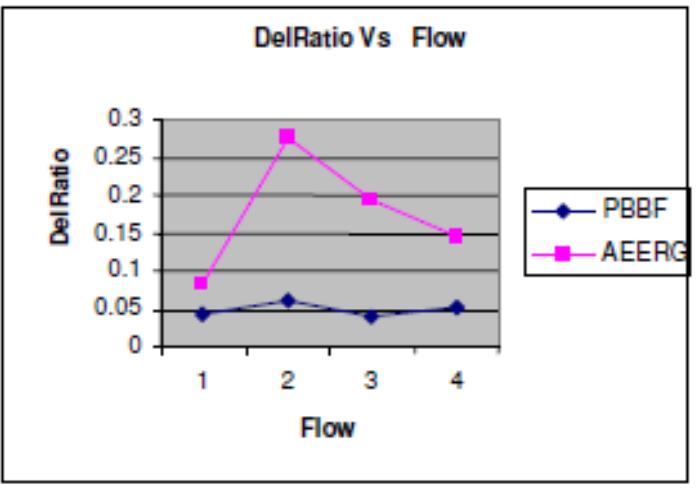

Fig. 4 Delivery Ratio Vs Flow

2) Based On Pvalue

In the second experiment, we vary the $\mathrm{p}$ values as $0.0,0.1$, $0.2,0.3$ and 0.4 .

Fig 5 gives the throughput of both the protocols when the number of flow (number of neighbor nodes which are receiving) is increased. As we can see from the figure, the 
throughput is more in the case of AEERG than PBBF.

Fig 6 shows the results of energy consumption for the number of flows $1,2,3,4$. From the results, we can see that AEERG scheme has less energy than PBBF scheme, since it has the energy efficient routing.

From Fig 7, we can ensure that the packets dropped are less for AEERG when compared with PBBF.

Fig 8 presents the packet delivery ratio of both the protocols. Since the packet drop is less and the throughput is more, AEERG achieves better delivery ratio, compared to PBBF protocol.

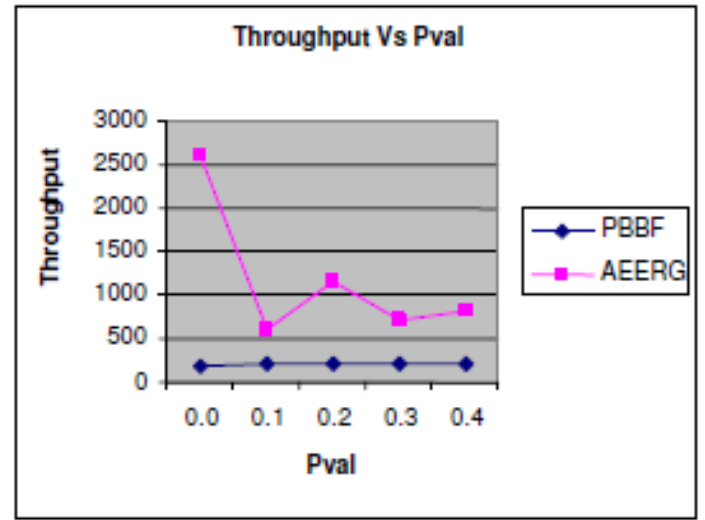

Fig. 5 Throughput Vs Pvalue

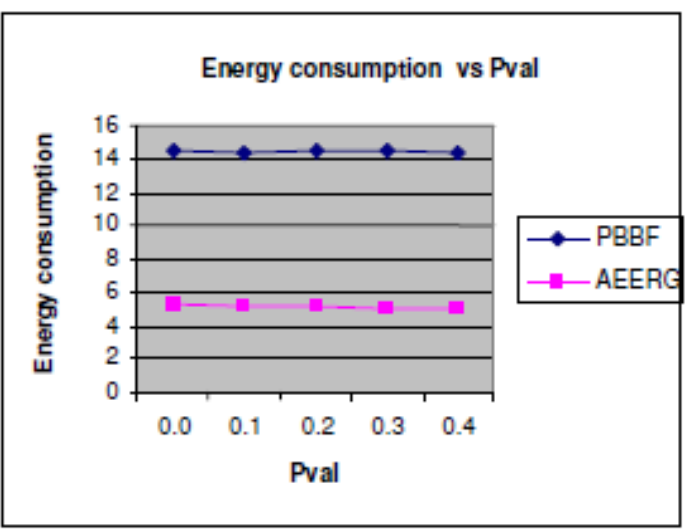

Fig. 6 Energy consumption Vs Pval

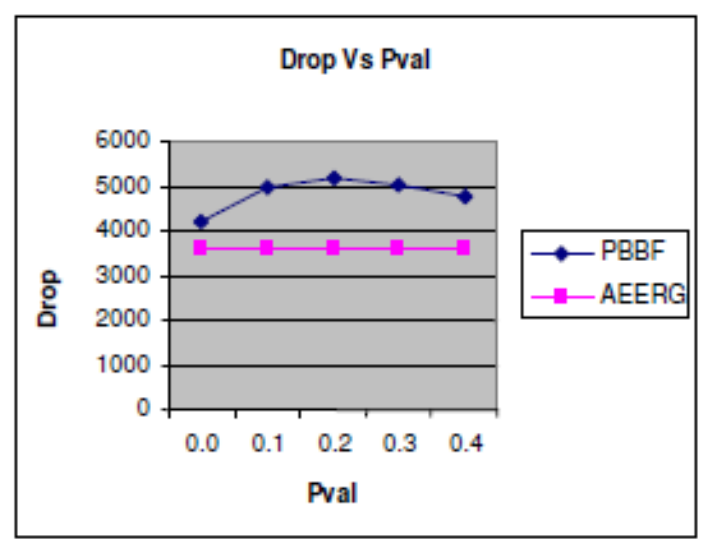

Fig. 7 Drop Vs Pval

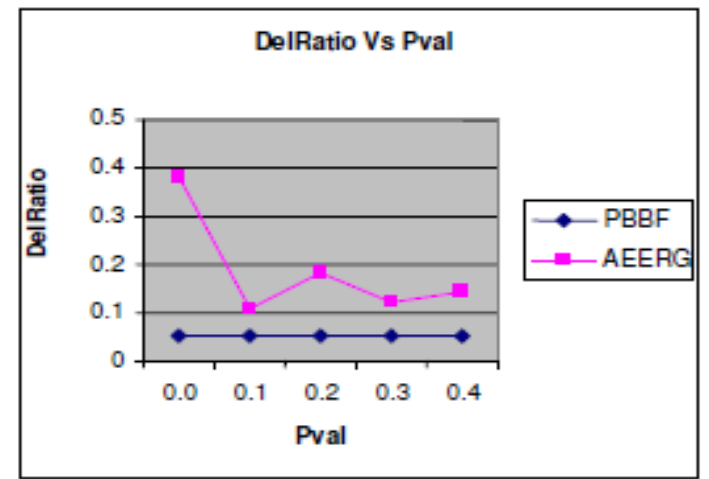

Fig. 8 Delivery Ratio Vs Pval

3) Based On Rate

In the third experiment, we vary the data sending rate as $100,200,300,400$ and $500 \mathrm{~kb}$.

Fig 9 gives the throughput of both the protocols when the number of flow is increased. As we can see from the figure, the throughput is more in the case of AEERG than PBBF.

Fig 10 shows the results of energy consumption for the number of flows $1,2,3,4$. From the results, we can see that AEERG scheme has less energy consumption than PBBF scheme, since it has the energy efficient routing.

From Fig 11, we can ensure that the packets dropped are less for AEERG when compared with PBBF.

Fig 12 presents the packet delivery ratio of both the protocols. Since the packet drop is less and the throughput is more, AEERG achieves good delivery ratio, compared to PBBF protocol.

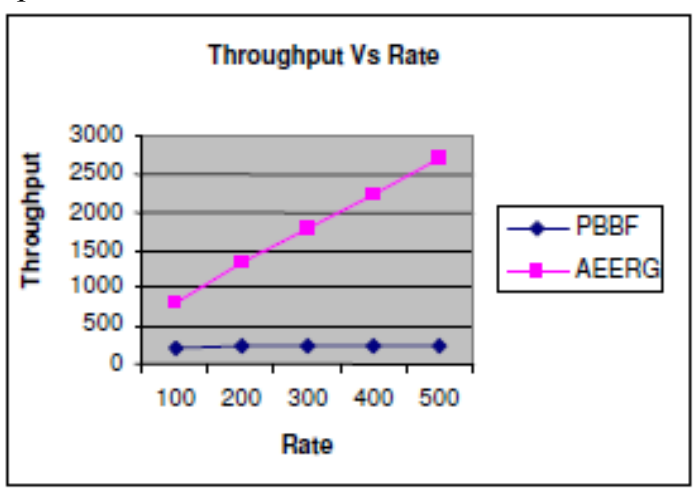

Fig. 9 Throughput Vs Rate

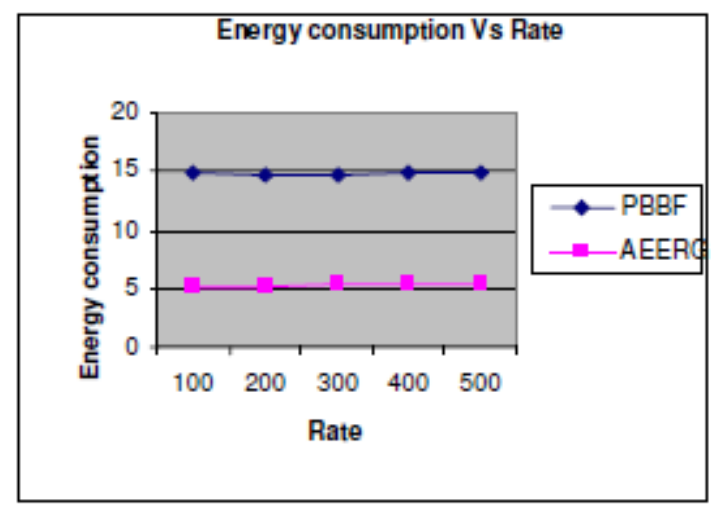

Fig. 10 Energy consumption Vs Rate 


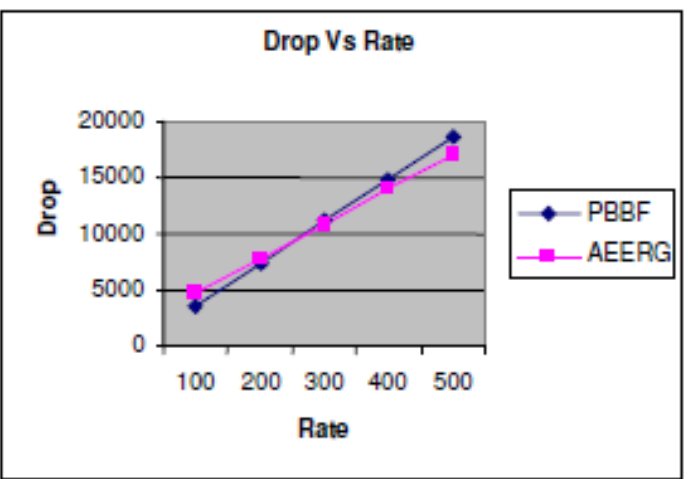

Fig. 11 Drop Vs Rate

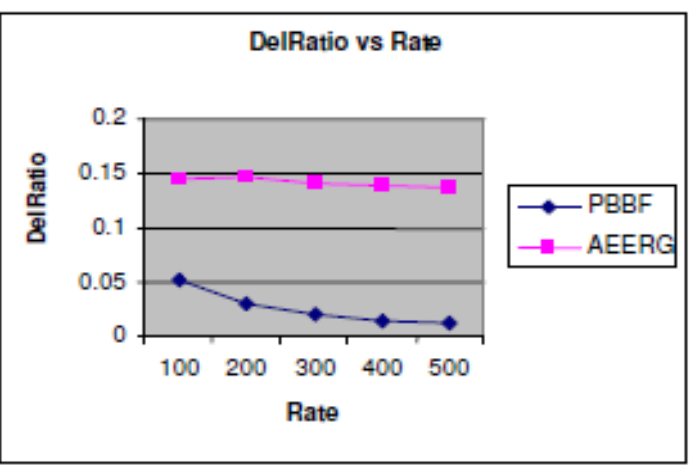

Fig. 12 DelRatio vs Rate

\section{CONCLUSION}

In this paper, we have proposed an Adaptive Energy Efficient and Reliable Gossip Routing Protocol. The main advantage of this protocol is that it overcomes the drawbacks of the existing protocols. This protocol assures the increased delivery ratio, better reliability and low energy consumption for gossip based routing. By simulation results, we have shown that the proposed protocol achieves good delivery ratio and good throughput with less packet drop and energy consumption. For further enhancement of this work, we plan to optimize the delay and overhead involved in the protocol.

\section{REFERENCES}

[1] Luo Junhai, Xue Liu, Ye Danxia," Research on multicast routing protocols for mobile ad-hoc networks", Elsevier, 2007

[2] Jing Xie, Luis Girons Quesada and Yuming Jiang," A Threshold-based Hybrid Routing Protocol for MANET"

[3] Xiaobing Hou and David Tipper," Gossip-based Sleep Protocol (GSP) for Energy Efficient Routing in Wireless Ad Hoc Networks", IEEE, March 2004

[4] Mike Burmester, Tri Van Le and Alec Yasinsac," Adaptive gossip protocols: managing security and redundancy in dense ad hoc networks" Elsevier Science Publishers, 2007.

[5] Yuval Shavitt and Amir Shay," Optimal Routing in Gossip Networks", IEEE, July 2005.

[6] Zhongmin Shi and Hong Shen," Adaptive Gossip-based Routing Algorithm", IEEE, 2004.

[7] Zygmunt J. Haas, Joseph Y. Halpern, and Li (Erran) Li," Gossip-Based Ad Hoc Routing", IEEE, 2006.

[8] Hany Morcos Ibrahim Matta Azer Bestavros," M2RC: Multiplicative-increase/additive-decrease Multipath Routing Control for Wireless Sensor Networks", ACM, 2004.

[9] Matthew J. Miller Cigdem Sengul Indranil Gupta," Exploring the Energy-Latency Trade-off for Broadcasts in Energy-Saving Sensor Networks", IEEE, 2008.

[10] Xiang-yang li, Kousha Moaveninejad and Ophir Frieder," Regional Gossip Routing for Wireless Ad Hoc Networks", Kluwer Academic Publishers, 2005 .

[11] Charles E. Perkins, Pravin Bhagwat, "Highly Dynamic Destination-Sequenced Distance-Vector Routing (DSDV) for Mobile Computers", Association of Computing Machinery, 1994.

[12] David B. Johnson, David A. Maltz, "Dynamic Source Routing in Ad Hoc Wireless Networks", Kluwer Academic Publishers, 1996.

[13] Charles E.Perkins, Elizabeth M.Royar, "Ad_hoc On_Demand Distance Vector Routing".

[14] T. Clausen, P. Jacquet, "Optimized Link State Routing Protocol (OLSR)", Network Working Group, October 2003.

[15] Vincent D. Park, M. Scott Corson, "A Highly Adaptive Distributed Routing Algorithm f or Mobile Wireless Networks", IEEE, 1997.

[16] Network Simulator, http://www.isi.edu.nsnam/ns

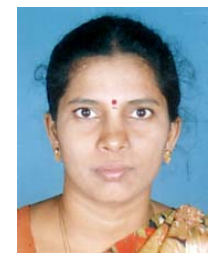

S.Rajeswari obtained her B.E. in Electronics and Communication Engineering (Bharathiyar university of Coimbatore, 1991), M.Tech in Communication Systems (National Institute of Technology, Trichirapalli, 2004) She is currently doing her research work on ad hoc networks which includes Routing Protocols, Power management and Reliability.

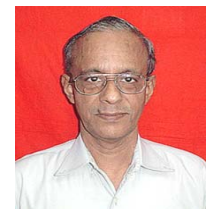

Dr.Y.Venkataramani obtained his B.Tech \& M.Tech from I.I.T Chennai. He received his Ph.D. from I.I.T Kanpur. He has served as a faculty for 34 years at NIT, Calicut and retired as Head of the Department. He served as Prinicipal of Saranathan College of Engineering, Trichy from 2001 to 2009. At present he is working as Director (Academic) in the same institution and PG Professor. His areas of interest include Computer Networks, Speech Processing and Image Processing 